Title:

CORONAL STRUCTURE INFERRED FROM REMOTE SENSING OBSERVATIONS

Author(s):

William C. Feldman

RECENED

JUL 19 19SS

OSTI

Submitted to

Scientific Basis for Robotic Explorations Close to the Sun

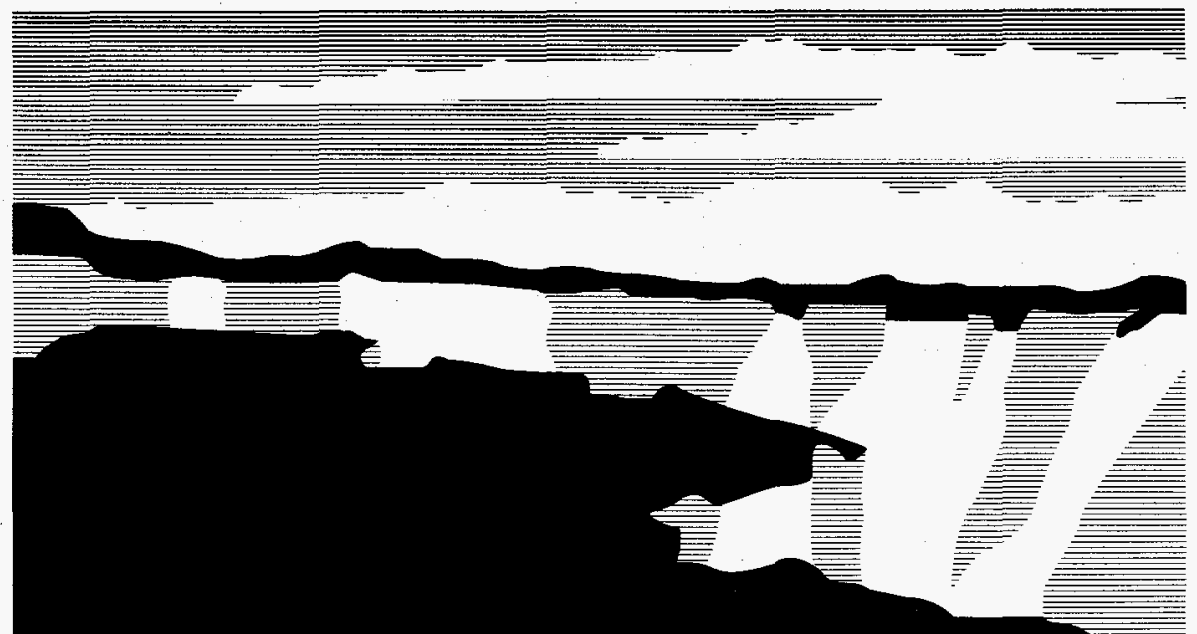

Los Alamos National Laboratory, an affirmative action/equal opportunity employer, is operated by the University of California for the U.S. Department of Energy under contract W-7405-ENG-36. By acceptance of this article, the publisher recognizes that the U.S. Government retains a nonexclusive, royalty-free license to publish or reproduce the published form of this contribution, or to allow others to do so, for U.S. Government purposes. The Los Alamos National Laboratory requests that the publisher identify this article as work performed under the auspices of the U.S. Department of Energy. 


\section{DISCLAIMER}

Portions of this document may be illegible in electronic image products. Images are produced from the best available original document. 


\section{DISCLAIMER}

This report was prepared as an account of work sponsored by an agency of the United States Government. Neither the United States Government nor any agency thereof, nor any of their employees, makes any warranty, express or implied, or assumes any legal liability or responsibility for the accuracy, completeness, or usefulness of any information, apparatus, product, or process disclosed, or represents that its use would not infringe privately owned rights. Reference herein to any specific commercial product, process, or service by trade name, trademark, manufacturer, or otherwise does not necessarily constitute or imply its endorsement, recommendation, or favoring by the United States Government or any agency thereof. The views and opinions of authors expressed herein do not necessarily state or reflect those of the United States Government or any agency thereof. 


\title{
Coronal Structure Inferred from Remote Sensing Observations
}

\author{
William C. Feldman \\ Los Alamos National Laboratory, MS D466 \\ Los Alamos NM 87545
}

\begin{abstract}
Remote-sensing observations of the Sun and inner heliosphere are reviewed to appraise our understanding of the mix of mechanisms that heat the corona and accelerate the solar wind. An assessment of experimental uncertainties and the basic assumptions needed to translate measurables into physical models, reveals very large fundamental uncertainties in our knowledge of coronal structure near the Sun. We develop a time-dependent, filamentary model of the extended corona that is consistent with a large number of remote sensing observations of the solar atmosphere and the solar wind.
\end{abstract}

\section{INTRODUCTION}

Although we have known for more than 50 years that the Sun's corona is more than ten times hotter than its photosphere ${ }^{1}$, we still do not know the mix of nonthermal mechanisms that supply the necessary energy. This gap in our knowledge exists despite a large effort devoted to this task (see, e.g., $/ 2 /$, and references therein). The central problem is that our entire data base rests on remote sensing observations, which do not have unique interpretations regarding details of coronal structure. Nevertheless, much is generally known from a combination of observational constraints, augmented by theory. These observations have included both scattered and directly emitted photons from the extended solar atmosphere, and in situ particles and fields observations measured beyond $0.3 \mathrm{AU}$. No data from in situ measurements of coronal structure exist at distances within $0.3 \mathrm{AU}$ of the Sun.

The solar corona as viewed in white light or soft $\mathrm{X}$ rays appears to be composed of streamers that extend outward from helmet-like closed-field structures. Coronal holes, defined by a very low rate of photon emission, fill the space between streamers. This quiescent state is disrupted at times by coronal mass ejections (CMEs), defined by the sudden expulsion of large volumes of relatively dense gas.

The outer portion of the corona as viewed through in situ observations of the solar wind, has three distinct states: the slow-speed and high-speed solar wind, and CMEs. Whereas slow-speed solar wind appears to originate in the generally magnetically closed corona, high-speed solar wind originates in coronal holes. CMEs as identified by several intrinsic characteristics in interplanetary space have been shown to be extensions of CMEs seen closer to the Sun through their transient enhanced photon emission ${ }^{3}$.

Interpretations of the solar wind beyond $0.3 \mathrm{AU}$ have identified the slow solar wind and CMEs as most-probably driven by time-dependent, spatiallystructured processes in the low corona $\mathrm{a}^{3,4}$, and the high-speed solar wind as driven by quasi-time-stationary, structure-free coronal processes ${ }^{5-7}$. By structure-free we mean that the time dependences of heating processes that drive the wind are much less than the convection times over coronal distance scales, needed to fill the overlying layers with smoothly outflowing gas.

We presently do not understand the mechanisms that supply the energy needed to maintain any of the foregoing classes of coronal structures. However, 
we expect the task of determining those that heat coronal holes and drive the highspeed solar wind to be the simplest, because of their nearly structure-free, timestationary appearance. Regardless of simplicity though, coronal holes and their high-speed extensions are important because they fill most of interplanetary space ${ }^{8-}$ ${ }_{10}$. We will therefore concentrate here on what is known, and needs to be known, about the state of the solar corona that supports a high-speed solar wind.

Our approach will build directly on properties of coronal gas at heliocentric distances less than 5.5 solar radii $\left(R_{s}\right)$ and beyond $0.3 \mathrm{AU}$, as inferred from a wide variety of remote-sensing observables. Rather than develop a single model that seems to be consistent with many of these observables (as done, e.g., by /11,12/), we will attempt to illustrate the range of conditions that are consistent with the observables as directly measured, but subject to different, yet plausible interpretations. Previous such attempts assumed a steady-state expansion that continuously fills the corona near the Sun ${ }^{11,13-17}$. If correct, then the fluid mass conservation equation,

$$
\mathrm{N}(\mathrm{R}) \mathrm{V}(\mathrm{R}) \mathrm{A}(\mathrm{R})=\mathrm{NeV}_{\mathrm{e}} \mathrm{A}_{\mathrm{e}}
$$

can be used to determine $V(R)$ given: 1) the solar wind particle flux measured at Earth, $\left.N_{e} V_{e}, 2\right)$ the areal expansion factor of individual stream tubes relative to that at Earth, $A_{e} / A(R)$, and 3) the profile of coronal densities, $N(R)$, inferred from coronagraph observations.

Once $V(R)$ is known, the radial dependence of total pressure can be determined from the momentum equation,

$$
m_{p} M N V(d V / d R)=-d P / d R-N G M_{s} m_{p} M /\left(R^{2} R_{s}\right) \text {, }
$$

where $m_{p}$ is the mass of the proton, $M$ is the mean molecular weight of the gas, $P$ is the total pressure (which includes contributions from the thermal plasma, waves, and possibly other sources of momentum), $G$ is the gravitational constant, and $M_{s}$ is the mass of the Sun. We define an effective temperature, $T_{\text {eff }}$, through the relation, $\mathrm{P}=\mathrm{NkT}_{\text {eff }}$, where $\mathrm{k}$ is Boltzmann's constant.

\section{OBSERVABLES}

Density: The profile of density in the radial range between 1.16 and $5.5 R_{s}$ has been inferred from the measured polarized brightness of Thomson-scattered white light from the photosphere. Densities have been inferred under the assumption of an absence of fine-scale filamentation. Four such determinations at polar latitudes near $\Lambda=90^{\circ}$ are reproduced in Figure $1^{14-16,18,19}$. All curves are as given in the literature except that from Saito $/ 19 /$, which used his combined latitude-radial distance formula averaged between $\Lambda=80^{\circ}$ and $90^{\circ}$. Inspection of Figure 1 shows that three of the four profiles are closely similar, but differ markedly from that obtained by Saito at distances larger than about $2 R_{s}$. We will not use the Saito profile in our subsequent estimates of coronal conditions because it cannot supply the observed solar wind mass flux at $1 \mathrm{AU}^{13}$. Nevertheless, we note that the large discrepancy evident in Figure 1 either means that densities within coronal holes can vary with time, or underscores the potentially large uncertainties in the procedure used to translate polarized brightness measurements to plasma densities. Published uncertainties range between about $\pm 15 \%$ and $\pm 35 \%$ at distances between 1.16 and $5.5 \mathrm{R}_{\mathrm{s}}^{16}$. The remaining three profiles all agree within these uncertainties. 


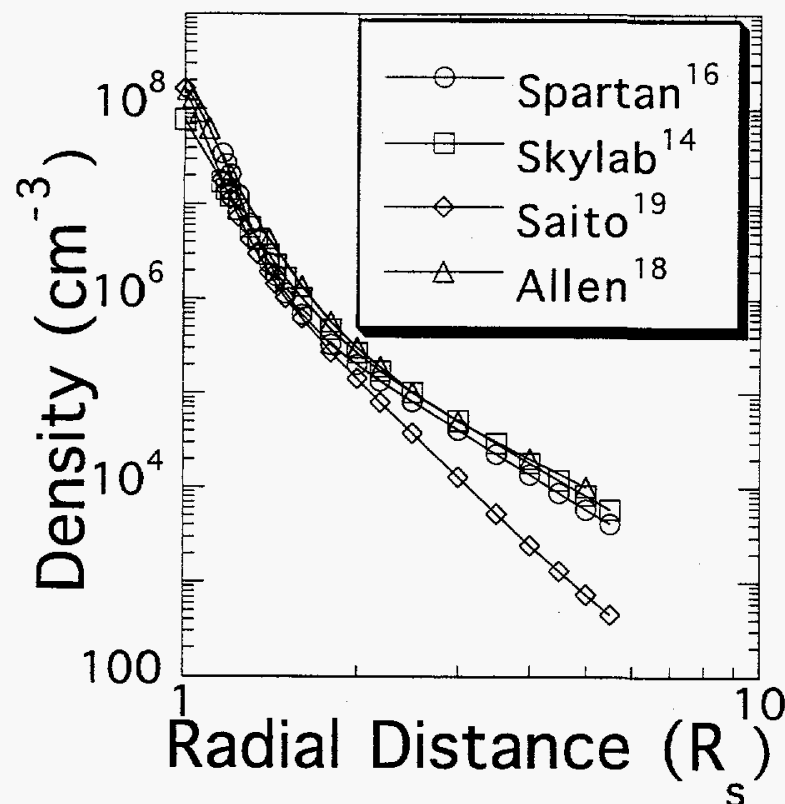

Figure 1. An overlay of radial profiles of coronal density determined from coronagraph data for four different coronal holes at polar latitudes.

It is not clear how much of these uncertainties refer to the absolute scale of densities and how much to their relative radial profile. We note though, that the profiles as published can all be fit with functions of the form, $N(R)=\sum a_{i} R^{-p_{i}}$, where $N(R)$ is the profile of electron density, $R$ is the heliocentric radial distance in $R_{s}$, and the $a_{i}$ and $p_{i}$ are constant coefficients ${ }^{15,17}$. We note further that for all three profiles, the power law index, $\mathrm{p}_{\mathrm{i}}$, at large distances is greater than $3^{17}$. Resultant large radial rates of density decrease indicate the need for continued acceleration out to $5.5 \mathrm{R}_{\mathrm{s}}$, or for non-stationary flow conditions ${ }^{17}$.

Magnetic Flux-Tube Topology: A second fundamental characteristic needed to specify the state of the corona (see Equation 1) is the non-radial divergence of the magnetic field, $\mathrm{F}_{\exp }(\mathrm{R})$, which defines the areal expansion along individual stream lines. Specifically,

$$
A(R)=R^{2} F_{e x p}(R),
$$

where $A(R)$ is the profile of flux-tube areas. This problem has been approached in several ways. One method is to model the most simple form of the corona, that observed at solar minimum, as a magnetic dipole augmented by an axisymmetric current sheet in its equatorial plane ${ }^{12}$. In this case,

$$
\mathrm{F}_{\mathrm{exp}}(\mathrm{R})=2 / \mathrm{R}+\mathrm{R}^{2} /\left[\mathrm{a}(\mathrm{a}+\mathrm{R})^{2}\right] \text {. }
$$

This model maps all of interplanetary space at large distances into both polar caps. If ' $a$ ' is determined by requiring the asymptotic value of $F_{\text {exp }}$ to equal that modelled during the polar passages of Ulyssess ${ }^{17,20}, F_{\text {exp }}(\infty)=10.4$, then ' $a$ '=5.2.

A second method is to model the boundaries of a polar hole and assume that all magnetic flux tubes within the hole expand homologously. This method was adopted for analysis of the polar hole observed by Skylab ${ }^{14}$, yielding,

$$
F_{\exp }(R)=F_{\max }\left\{\exp \left[\left(R-R_{1}\right) / \sigma\right]+F_{1}\right\} /\left\{\exp \left[\left(R-R_{1}\right) / \sigma\right]+1\right\}
$$


where $F_{1}=1-\left(F_{\max }-1\right) \exp \left[\left(R_{0}-R_{1}\right) / \sigma, F_{\max }=7.26, R_{1}=1.31, R_{0}=R_{s}\right.$, and $\sigma=0.51$.

A last method is to adopt a combined source-surface and current-sheet model of the corona $a^{20}$. This model is fully specified by the boundary condition at the coronal base given by the photospheric magnetic field measured by the Wilcox solar Observatory (WSO), and by adoption of a current sheet that follows the observed magnetic equator of the Sun. The resultant radial profile at polar latitudes calculated during the time of the Ulysses polar passage, can be modelled by ${ }^{17}$,

$$
F_{\text {exp }}(R)=[10.4 \exp (R / D)-8.4 \exp (1 / D)] /[\exp (R / D)+\exp (1 / D)] \text {. }
$$

Selected profiles are compared in Figure 2. Their differences translate directly into uncertainties in the coronal flow state through use of Equations 1 and 2.

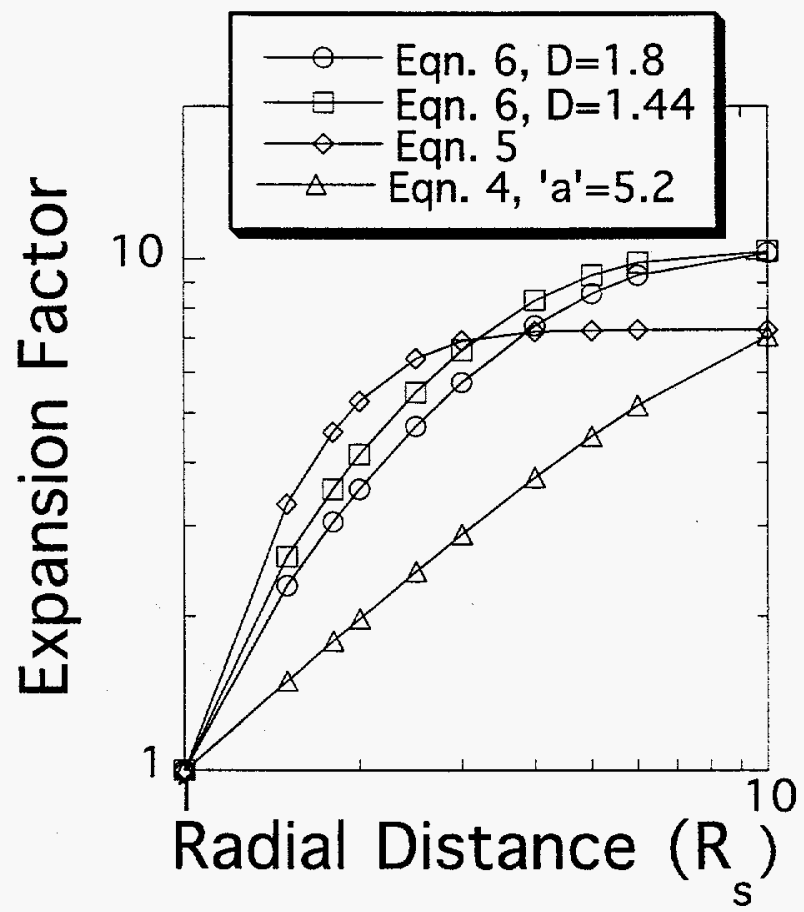

Figure 2. An overlay of four models of the non-radial expansion factor of polar magnetic flux tubes. The curves marked by circles and squares are fits to the mean profile of Wang et al. ${ }^{20}$ calculated when Ulysses was poleward of $\pm 60^{\circ}$ heliographic latitude. The profile estimated by Munro and Jackson ${ }^{14}$ is marked by diamonds, and that for the McKenzie et al. ${ }^{12}$ model is marked by triangles.

Temperatures at the Base of the Corona: Temperatures of electrons in coronal holes have been inferred from a variety of remote sensing observations ${ }^{21,22}$. Although most observations can be reconciled with a single, spatially uniform temperature that is less than $1.3 \times 10^{6} \mathrm{~K}$ for $\mathrm{R}<1.6^{21}$, analysis of Yohkoh SXT soft X-ray data yields temperatures in the range between 1.8 and $2.4 \times 10^{6} \mathrm{~K}$ at distances less than $1.1 \mathrm{R}_{\mathrm{s}}^{22}$. One possible resolution to this discrepancy is that plasma in coronal holes near its base is not spatially uniform. Instead of a single structure-free medium, it may consist of an ensemble of thin filaments having different plasma states. It is of course also possible that this discrepancy results from uncertainties that are traceable to interpretations of different observables. We note though that observations of line shapes of $\mathrm{Ly} \alpha$ at $1.6 \mathrm{R}_{\mathrm{s}}$ indicate twocomponents having temperatures of $1.6 \times 10^{6} \mathrm{~K}$ and $7.6 \times 10^{6} \mathrm{~K}$, respectively ${ }^{23}$.

Inferred Flow State in Coronal Holes Near the Sun: The recent polar traverse of Ulysses $^{10}$, and measurements of the white-light corona by Spartan-201 ${ }^{16}$, has provided the observational underpinnings necessary to use Equations 1 and 2 to solve for profiles of solar wind speed and pressure near the Sun. At the time of 
this traverse, the solar cycle was approaching minimum conditions, the solar corona had developed the appearance of a simple dipole augmented by a planar current sheet that paralleled the Sun's rotational equatorial plane, and both poles of the Sun were covered by large-area coronal holes. However, before proceeding to solve Equations 1 and 2, we first need to resolve an essential ambiguity in interpretation of the observables. Is the gas that resides in the corona within coronal holes smoothly distributed (having plasma characteristics that do not vary sharply in space or time), or is coronal structure composed of an ensemble of many thin filaments that are filled with transient jets of relatively dense, fast-moving gas?

Discrimination between these extremes is not possible from remote-sensing observations because they necessarily integrate over a large volume of gas along the line of sight. Even though the polar corona generally appears to be relatively free of fine-scale structure, many auxiliary observations suggest existence of nonstationarity and/or fine-scale filamentation; 1) Jets of $\sim 10^{5} \mathrm{~K}$ gas generated by explosive events are observed on every field line open to the corona at rates between 7.5 and 15 times a day ${ }^{17,24}$. Additional, apparently unrelated jet-like phenomena seen at $\sim 10^{5} \mathrm{~K}$ are macrospicules ${ }^{25,26}$. 2) Jets of $\sim 2 \times 10^{6} \mathrm{~K}$ gas are seen in soft $\mathrm{X}$ rays to originate at bright points both within and outside of coronal holes, and extend upward to altitudes as high as $4 \times 10^{5} \mathrm{~km}^{27}$. 3) High-pass-filtered pictures of the polar corona in white light show striations having angular widths that match the diameters of supergranules ${ }^{16,28}$. These striations have been associated with polar plumes, which have a lifetime of hours to days ${ }^{29}$. 4) Spectra of Ly $\alpha$ in Coronal holes reveal two superimposed components $\left(1.6 \times 10^{6} \mathrm{~K}\right.$ and $\left.7.6 \times 10^{6} \mathrm{~K}\right)^{23}$. 5) Structure functions of scintillations of radio waves from sources occulted by the solar corona between about 2 and $6 R_{s}$ reveal density striations that have large axial ratios aligned with the heliocentric radial ${ }^{30}$. The apparent speeds of coherent patterns of these fluctuations range between 400 and $1280 \mathrm{~km} \mathrm{~s}^{-1} 3 \mathrm{f}$. And 6) velocity distributions of protons throughout the high-speed solar wind beyond $0.3 \mathrm{AU}$ reveal two relatively convecting, interpenetrating components ${ }^{32-34}$.

Because it is not possible to decide between the foregoing alternatives from existing remote-sensing observables, both assumed states need to be given equal credibility at the outset. Such an analysis was conducted recently ${ }^{17}$. Inversion of Equation 1 to deterimine the speed profile near the Sun under the assumption of a spatially and temporally uniform corona is relatively straightforward. Sample results for non-radial expansion factors given by Equations 4 and 6 are shown in Figure 3. They differ markedly throughout the radial distance range that is shown, although both profiles have the same asymptotic expansion factor, 10.4. Indeed, their difference amounts to more than a factor of three for $\mathrm{R}$ between 2 and 5.5.

Derivation of speed profiles under the assumption of an intrinsically filamented corona consisting of spatially-discrete jets of relatively dense, fast plasma, is given in $/ 17 \%$. Each jet is assumed to be separated by relatively slow and low density gas from the ambient solar wind and/or the rarefaction region generated from evolution of the trailing part of the previous jet. A representative speed profile determined from Equation 1 is shown in Figure 3. Inspection shows it to have a very different character than either of the two steady-state profiles. Rather than increasing monotonically with increasing radial distance, it maximizes at $\sim 1300 \mathrm{~km} \mathrm{~s}^{-1}$ at $\sim 1.5$ $R_{s}$ before decreasing to a constant terminal speed (at $5.5 R_{s}$ ) of $\sim 780 \mathrm{~km} \mathrm{~s}^{-1}$. When one considers the very large uncertainties in determining this speed profile from measured observables using Equation 1, it can only be fortuitous that this speed is very close to that measured by Ulysses, $\mathrm{V}=770 \pm 23 \mathrm{~km} \mathrm{~s}^{-1}$.

Given the foregoing speed profiles, one can proceed to estimate the profile of plasma pressure, and hence an effective temperature, from Equation 2. Because this equation was derived under the explicit assumption of time stationarity, it can 


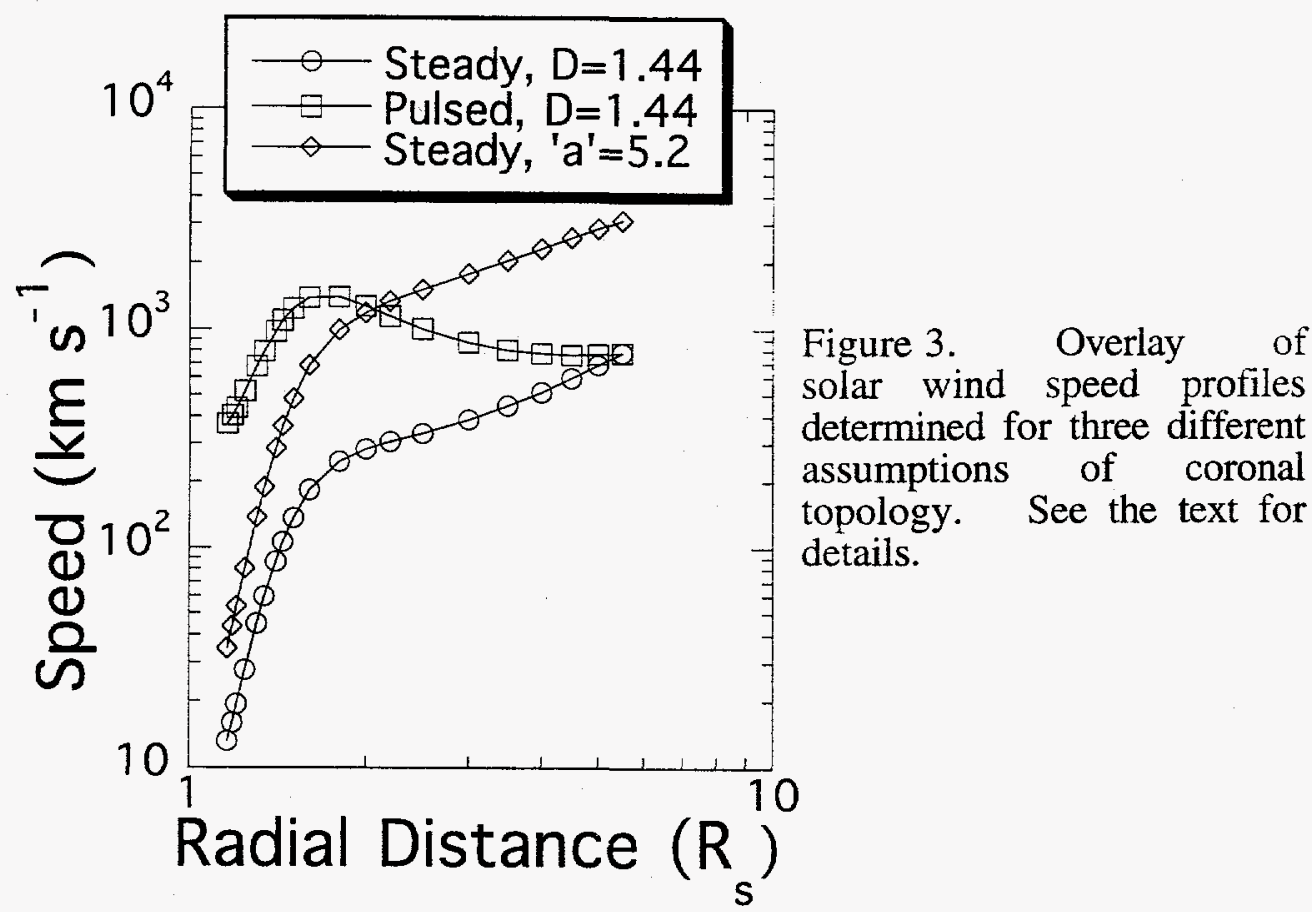

only be applied for the steady-state model of the polar corona. The result is prediction of a broad distribution of very hot effective temperatures, approaching a maximum close to $10^{7} \mathrm{~K}$ at about $4 \mathrm{R}_{\mathrm{s}}{ }^{17}$. Such high extended temperatures require extended heat and/or momentum input to the accelerating plasma.

\section{SUMMARY AND DISCUSSION}

Summary of Results: Models of the coronal flow state were derived from representative sets of remote-sensing data using two extreme, yet credible assumptions. The first assumed a steady-state, spherically symmetric flow that fills the corona near the Sun. Resultant profiles of flow speed are monotonically increasing out to, and perhaps beyond, $5.5 \mathrm{R}_{\mathrm{s}}$, but differ markedly in magnitude because of large uncertainties in both the observables and their analysis procedures. All such profiles require extended energy and/or momentum additions in order to maintain an extended positive acceleration.

An alternative assumption is that the corona is filamentary and time dependent. Such an assumption is supported by a host of remote-sensing observations summarized in section 2 . Its adoption yields a speed profile that is very different from that which results from a steady-state model using the same data. In contrast to the monotonic acceleration of plasma out to at least $5.5 R_{s}$, the speed profile reaches a maximum at $\sim 1.5 R_{s}$, and then decreases to a constant value at $5.5 R_{s}$. Of course, all speed profiles between the steady-state and pulsed-model profiles shown in Figure 3 are possible if only a fraction of the coronal magnetic flow tubes map to supergranule cells that support reconnection driven transients.

Derivation of the pressure profile for the pulsed model is not simple because an explicitly time-dependent hydrodynamic analysis is required.

Discussion: A schematic picture of an assumed filamentary, time-dependent corona has been assembled ${ }^{17}$ from a host of remote-sensing observables. The resultant picture is shown in Figure 4. It is composed of an ensemble of $2.5^{\circ}$-wide flow tubes extending between the photosphere and $\sim 14 R_{s}$. The radial segments on 


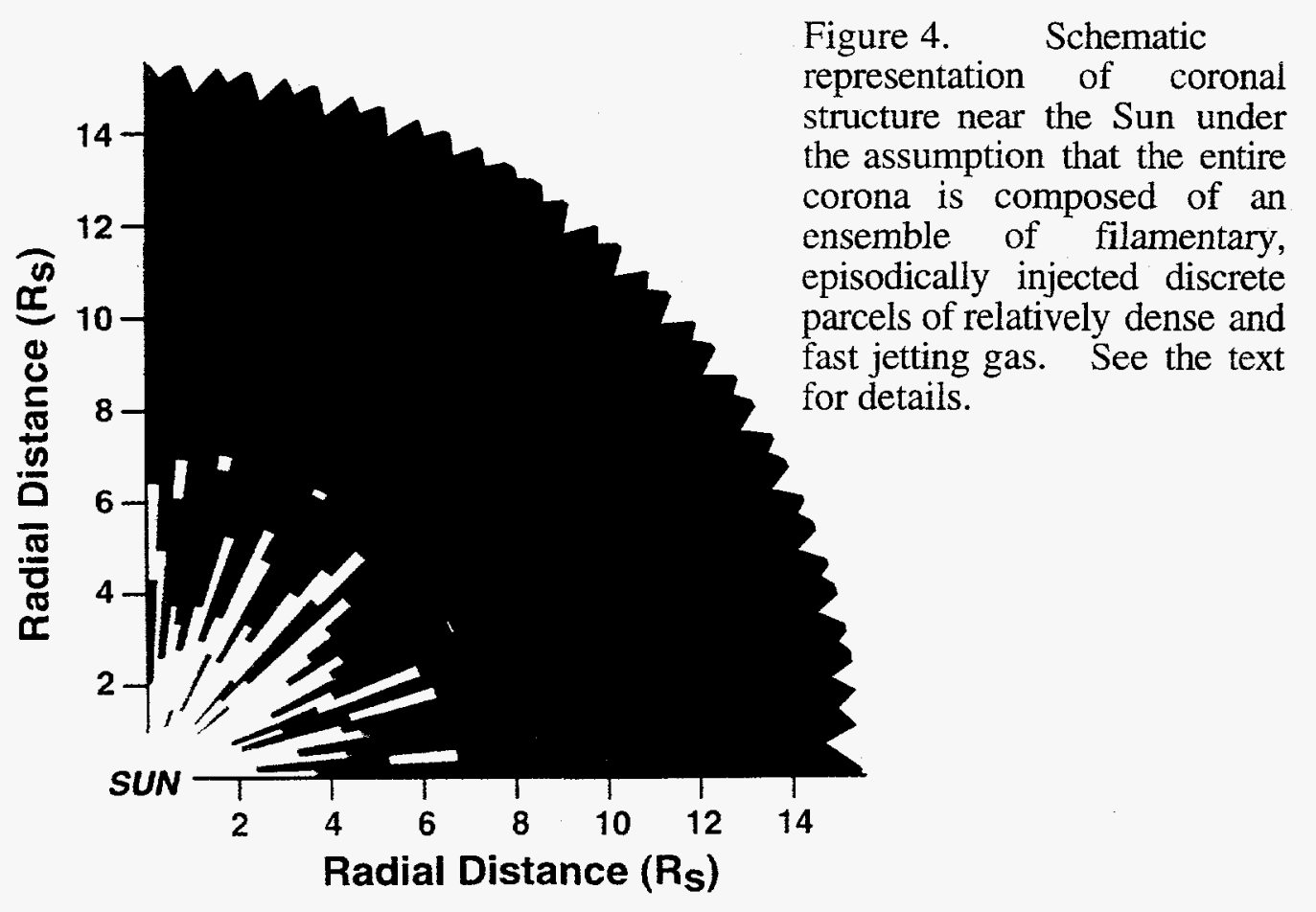

each flow tube that correspond to the most recently launched jets are colored red, those that correspond to the previously launched jets are colored blue, and those that correspond to an overlap between two jets are colored green. The remaining segments are left white.

Inspection reveals several interesting observations. First, there are a lot of 'empty' flow-tube segments between discrete jets at radial distances sunward of 6 $R_{s}$, but very few such segments beyond $6 R_{s}$. Surface currents must flow along all boundaries between the jet segments and their neighboring low density 'empty' segments, in order to confine the jets to their flow tubes by $\mathbf{J} X \mathbf{B}$ forces. However, these currents are unstable to the generation of electrostatic drift waves having $\mathbf{k}$ vectors perpendicular to $\mathbf{B}$ and wavelengths of the order of the proton gyroradius $^{35}$. Such a configuration is consistent with IPS observations ${ }^{30,31,36}$. It provides a natural (and to our knowledge, the only) explanation for their observed radial profile (i.e., the axial ratios of IPS density irregularities decrease sharply from values greater than 10 at distances less than $6 R_{s}$, to unity at larger distances). Resultant density gradients evident in Figure 4 should provide conditions that are prime for the enhanced damping of Alfvén waves through application of a host of non-standard processes summarized by Parker ${ }^{37}$.

We also note from Figure 4 that interactions between the leading edges of succeeding jets and the trailing edges of preceeding jets begin beyond $\sim 6 R_{s}$, where the flow is collisionless yet still sub-Alfvénic. A very complex interaction should develop, perhaps involving the generation of strong forward and reverse electrostatic shocks. This interaction should have measurable consequences at radial distances well beyond $14 R_{s}$. A determination of this interaction and its consequences needs to be pursued.

Acknowledgments. This work was performed under the auspices of the U.S. Department of Energy with financial support from NASA. 


\section{References}

1. Billings, D.E., A Guide to the Solar Corona, Chaps. 1 and 3, Academic Press, San Diego (1966).

2. Narain, U., and P. Ulmschneider, Space Sci. Rev., 54, 377 (1990).

3. Gosling, J.T., Physics of Magnetic Flux Ropes, Geophys. Monogr. 58, eds. C.T. Russell, E.R. Priest, and L.C. Lee, Amer. Geophys. Union, 343, (1990).

4. Axford, W.I., J.F. McKenzie, Solar Wind Seven, eds. E. Marsch and R. Schwenn, Pergamon, Oxford, 1, (1992).

5. Feldman, W.C., J.R. Asbridge, S.J. Bame, and J.T. Gosling, J. Geophys. Res., $\underline{81}$, 5054, (1976).

6. Feldman, W.C., J.R. Asbridge, S.J. Bame, and J.T. Gosling, The Solar Output and its Variations, ed. O.R. White, Colo. Assoc. Univ. Press, Boulder, 351, (1977).

7. Bame, S.J., J.R. Asbridge, W.C. Feldman, and J.T. Gosling, J. Geophys. Res., $\underline{82}$, 1487, (1977).

8. Bohlin, J.D., Coronal Holes and High Speed Solar Wind Streams, ed. J.B. Zirker, Colo. Assoc. Univ. Press, Boulder, 27, (1977).

9. Gosling, J.T., S.J. Bame, W.C. Feldman, et al., Geophys. Res. Lett., 22, 3329, (1995).

10. Phillips. J.D., S.J. Bame, A. Barnes, et al., Geophys. Res. Lett., 22, 3301, (1995).

11. Habbal, S.R., R. Esser, M. Guhathakurta, and R.R. Fisher, Geophys. Res. Lett., 22, 1465, (1995).

12. McKenzie, J.F., M. Banazkiewicz, and W.I. Axford, Astron. Astrophys., 303, L45, (1995).

13. Feldman, W.C., J. Geophys. Res., 82, 667, (1977).

14. Munro, R.H., and B.V. Jackson, Astrophys. J., 213, 874, (1977).

15. Lallement, R., T.E. Holzer, and R.H. Munro, J. Geophys. Res., 91, 6751, (1986).

16. Fisher, R., and Guhathakurta, M., Astrophys. J., 447, L139, (1995).

17. Feldman, W.C., S.R. Habbal, G. Hoogeveen, and Y.-M. Wang, J. Geophys. Res., Subm., (1996).

18. Allen, C.W., Astrophysical Quantities, Athlone, London, 176, (1973).

19. Saito, K., Ann. Tokyo Astron. Observ., 12, 53, (1970).

20. Wang, Y.-M., S.H. Hawley, and N.R. Sheeley, Jr., Science, 271, 464, (1996).

21. Habbal, S.R., R. Esser, and M.B. Arndt, Astrophys. J., 413, 435, (1993)

22. Hara, H. S. Tsuneta, L.W. Acton, et al., Publ. Astron. Soc. Japan, 46, 493, (1994).

23. Kohl, J.L., L.D. Gardner, L. Strachan, et al., Space Sci. Rev., 72, 29, (1995).

24. Dere, K.P., Solar Wind Seven, eds. E. Marsch, R. Schwenn, Pergamon Press, Oxford, 11, (1992).

25. Bohlin, J.D., S.N. Vogel, J.D. Purcell, et al., Astrophys. J. Lett., 197, L133, (1975).

26. Withbroe, G.L., D.T. Jaffe, P.V. Foukal, et al., Astrophys. J., 2033, 528, (1976).

27. Shimojo, M., S. Hashimoto, K. Shibata, et al, Publ. Astron. Soc. Japan, 48, 123, (1996).

28. Koutchmy, S., Solar Phys., 51, 399, (1977).

29. Habbal, S.R., Ann. Geophysicae, 10, 34, (1992).

30. Armstrong, J.W., W.A. Coles, M. Kojima, and B.J. Rickett, Astrophys. J., 358, 685, (1990).

31. Klinglesmith, M.T., W.A. Coles, and R.R. Grall, (Abstract) EOS, F470, 1995.

32. Feldman, W.C., B. Abraham-Shrauner, J.R. Asbridge, et al., Physics of Solar Planetary Environments, ed. D.J. Williams, AGU, Wash., D.C., 413, (1976).

33. Feldman, W.C., J.T. Gosling, D.J. McComas, and J.L. Phillips, J. Geophys. Res., 98, 5593, (1993).

34. Marsch, E., K.-H. Muhlhauser, R. Schwenn, et al., J. Geophys. Res., 87, 52, (1982).

35. Gary, S.P., Theory of Space Plasma Microinstabilities, Cambr. Univ., Cambr., chapter 4, (1993).

36. Coles, W.A., W. Lui, J.K. Harmon, and C.L. Martin, J. Geophys. Res., 96, 1745, (1991).

37. Parker, E.N., Solar Wind Seven, eds. E. Marsch and R. Schwenn, Pergamon, Oxford, 79, (1992). 